\title{
Developing of the smart quadcopter with improved flight dynamics and stability
}

\author{
Waqas Malik ${ }^{1 *}$ and Sakhawat Hussain ${ }^{2}$
}

*Correspondence:

wmalik@ualberta.ca

${ }^{1}$ University of Alberta, 59127

Ave SW, Edmonton T6X0G5,

Canada

Full list of author information

is available at the end of the article

\begin{abstract}
The cause of the rapid growth and enhancement in the dynamics of unmanned aerial vehicles (UAVs) is due to its vast utilization in every-day application. The major advantage of UAVs is no risk of human life with secure and suitable surveillance. The UAV facilitates in live video streaming and wide aerial coverage for monitoring. In this project, a type of UAV named quadcopter has been developed. The work mainly consists of design of the quadcopter frame, interfacing of the brushless DC motors and bluetooth module with the microcontroller, and adjustment of the roll, pitch, and yaw for keeping the smooth flight dynamics by flight controller board containing MEMS sensors, i.e., gyroscope, accelerometer, magnetometer, and pressure sensors. The repeated simulation and testing has been carried out in MATLAB for the mathematical modeling of the dynamics of the system, i.e., Euler method for solving differential equation for finding system states, computation of the rotation matrix $R$ and functions to convert from an angular velocity vector $w$ to the derivatives of roll, pitch, and yaw. The improvement in the parameters of the flight dynamics and removing the errors to gain stability in the frame are the main core issues which have been achieved by the several iterations. A closed-loop control system, i.e., PID controller, has been simulated and carefully designed for stabilizing the actual angle from the sensors and desired angle from the pilot. The reduced error rate of 0.05 degrees after every $10 \mathrm{~s}$ was achieved.
\end{abstract}

Keywords: Quadcopter, Flight dynamics, MEMS sensors

\section{Introduction}

Compact air vehicles with rotor craft capable of vertical takeoff and landing are called quadcopters. These are similar to helicopter capable of hovering, and because of simple mechanical structure and ease of operating, their demand is increasing since they have no swashplate mechanism. The popularity of quadcopter has increased, and the first quadcopter was developed in 1920s [1,2]. The increasing demand of quadcopter $[3,4]$ is due to the research-grade platforms.

The designing and fabrication of quadcopter are challenging. It mainly consists of mathematical model in time and frequency space domain. The quadcopter [5, 6] mathematical model is a nonlinear model.

A quadcopter has four similar dispersed rotors, located at four corners of the squareshaped frame. The electronically operated rotors made it easier to control and hence adaptable for multiple applications. Quadcopter falls under the category of mobile robots with four control inputs having six degrees of freedom (DOF), in which three are 
translational and three are rotational. This rotational and translational movement makes it flexible in moving in any direction.

\section{Method}

The project is divided into different blocks. The microcontroller Atmel 16 is used to control the speed and PWM of the four brushless motors M1, M2, M3, and M4. The flight controller board MinIMU-9, which contains gyroscope, accelerometer, magnetometer, and pressure sensors, is used to adjust control variables raw, pitch, and yaw at every instant with complementary second-order high-pass filter. The bluetooth module HC 05 is powered by LIPO battery and serial communication between android-based applications to control the movements of the quadcopter. The purpose of using LIPO battery is because of its lighter weight and higher efficiency. The output of the PID control system is a PWM signal whose pulse width is varied to control the motor speed. MATLAB software is used to tune the PID control in real systems. Figure 1 shows the block diagram of the quadcopter.

\section{Quadcopter frame design}

During the design of the quadcopter frame, the most important factor to determine is the center point of gravitation [7] of the frame. To find the point, two tests were conducted. In suspension test, we tied four arms of the quadcopter to the string and place small weight and checked for the point where system gets perfectly aligned in the air. The weights were placed, and the orientation was noted by the sensors. Secondly, the tipping test was performed in that test the frame of the quadcopter was placed on the rigid pivot, and the point was selected that can withstand the whole of the frame body. In both of the above tests, orientation of the points was close to each other. After determining the point, it was decided to calculate the symmetric coefficient. These coefficients are required to stabilize the body in air. The PID controller [8, 9] was selected because of the ability to tune and handle the nonlinearity of the system at every instant. Tuning was done in MATLAB and repetitive flight testing of the frame has been done.

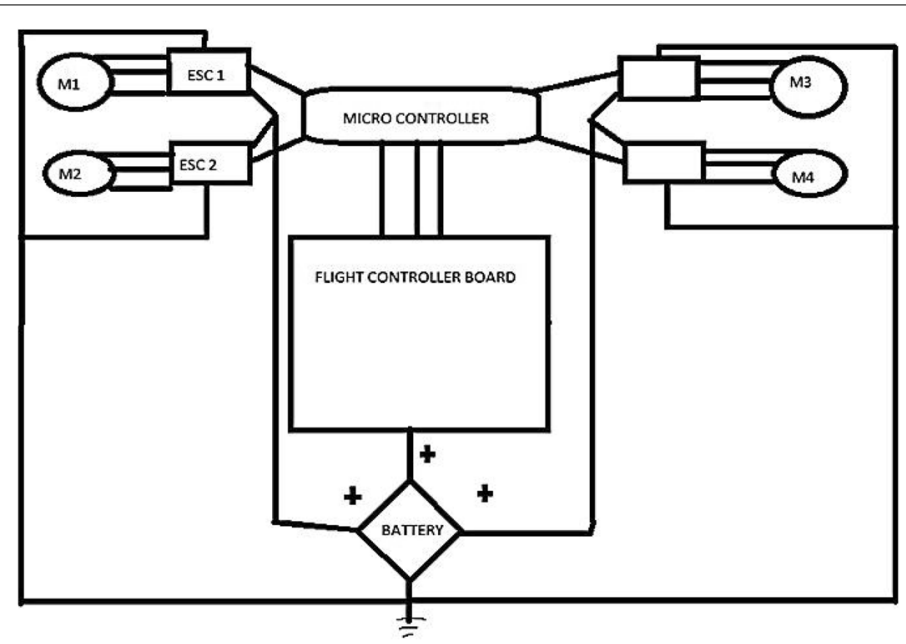

Fig. 1 Block diagram of quadcopter 
The final successful frame designed was made up of thermopore, which results in stability, and hence, collision issues were resolved.

\section{Flight controller board}

The flight controller board used here is mounted with MEMS sensors, i.e., gyroscope, accelerometer, magnetometer, and pressure sensors to control the variables roll, pitch, and yaw for a stable flight. MinIMU-9 board [10] used here is a perception sensor that perceives the information from the environment. This board is interfaced with the microcontroller to send and receive data. Block diagram of the communication is shown in Fig. 2.

\section{Brushless DC motors}

In this project, the brushless dc motors [11] are used. The value of the thrust per motor is calculated [11] by the following formula:

Thrust one each motor $=($ Total weight $\times 2) / 4$ Following is the reason for selecting these motors in this project.

- These motors are high-speed motors with the operating speed of $10000 \mathrm{rpm}$ either loaded or in unloaded condition.

- The inner rotor of the motor has low inertia which can be accelerated, decelerated, and can be moved in reverse direction which results in rapid acceleration and response time.

- These motor are capable of producing high running torque per cubic of a DC motor, which results in increased power density

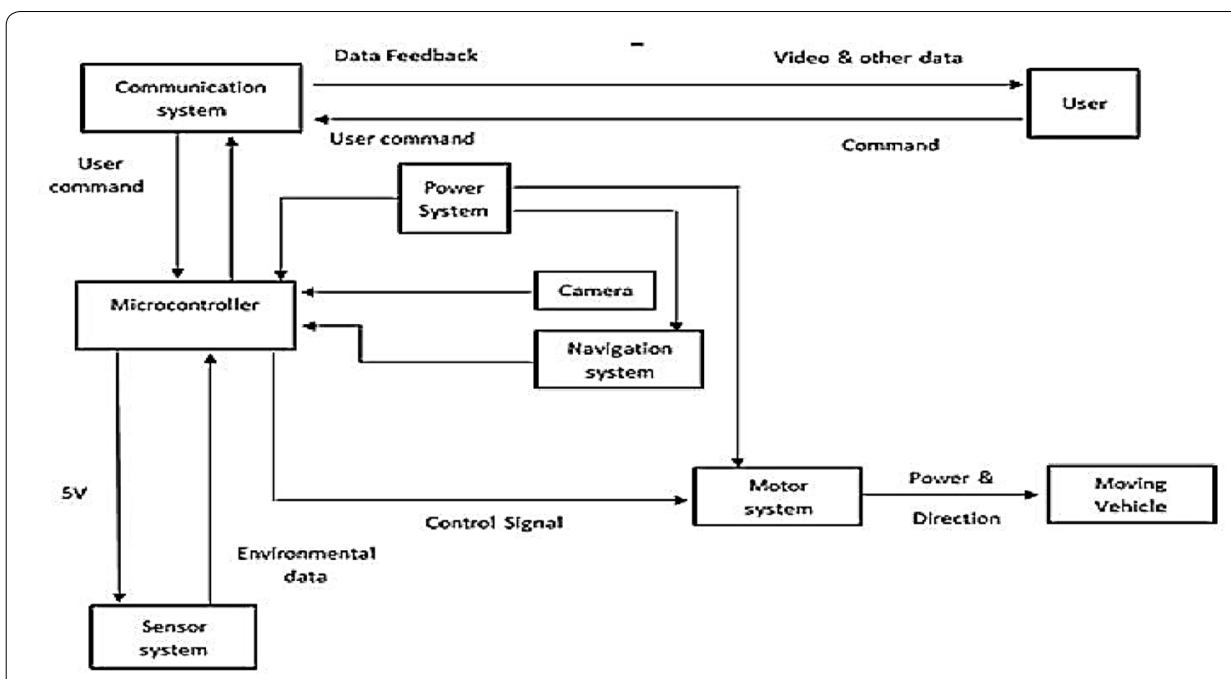

Fig. 2 Block diagram of the sensor system communication 
- The brushless motors have good lifetime and long running hours approximately $10000 \mathrm{~h}$ and hence less chances of replacing or repairing and are very reliable.

- Propeller Length and Pitch

In the quadcopter, there are four propellers, two rotating in clockwise and other two in the anticlockwise direction. The length and the pitch of the propeller greatly depend on the speed and stability of the quadcopter. The greater the length and the pitch more power will be used, hence more current will be drawn and vice versa. Therefore, I have used the propeller with shorter length and low pitch because of the stability of the frame during the flight time.

\section{PID controller tuning}

PID controller [8, 9] has been selected as the most suitable controller for the quadcopter. The tuning of the control parameter has been done by keeping in view the factors of the balancing the inertial moment and the generated force from the motors. The inertial moment depends on the total mass and motor properties such as payload. A closedloop control system, i.e., PID controller has been simulated and carefully designed for stabilizing the actual angle from the sensors and desired angle from the pilot. The fine reduced error rate of 0.05 degrees after every $10 \mathrm{~s}$ was achieved in the actually designed controller. Figure 3 shows the PID structure at each of the coordinate axis.

\section{Frame stability}

The controller used has been simulated/tuned several times to obtain the stability [12, $13]$ in the frame. The adjustment in the parameters of angular velocities and angular displacements [14] are taken into the account. The roll, pitches, and yaw angles in the body frame are $\theta=(\phi, \theta, \psi)$. The graph of the angular velocities and angular displacement with respect to the roll, pitch, and yaw angles $\phi, \theta$, and $\Psi$, respectively, are shown with red, green, and blue lines. The manually generated graphical plot of angular velocities versus time and angular displacement versus time is shown in Fig. 3.

The automatically generated simulation results of angular velocity and angular displacements were far better than the manually generated results. The automatically generated graphical plot of angular velocities versus time and angular displacement versus time is shown in Fig. 4.

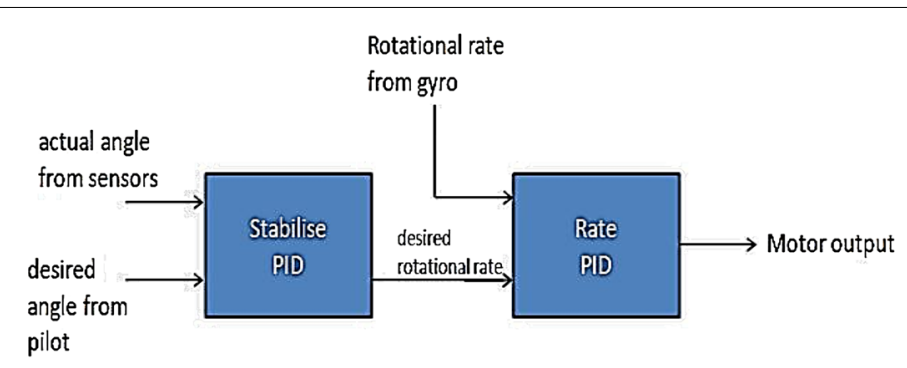

Fig. 3 PID structure at each of the coordinate axis 

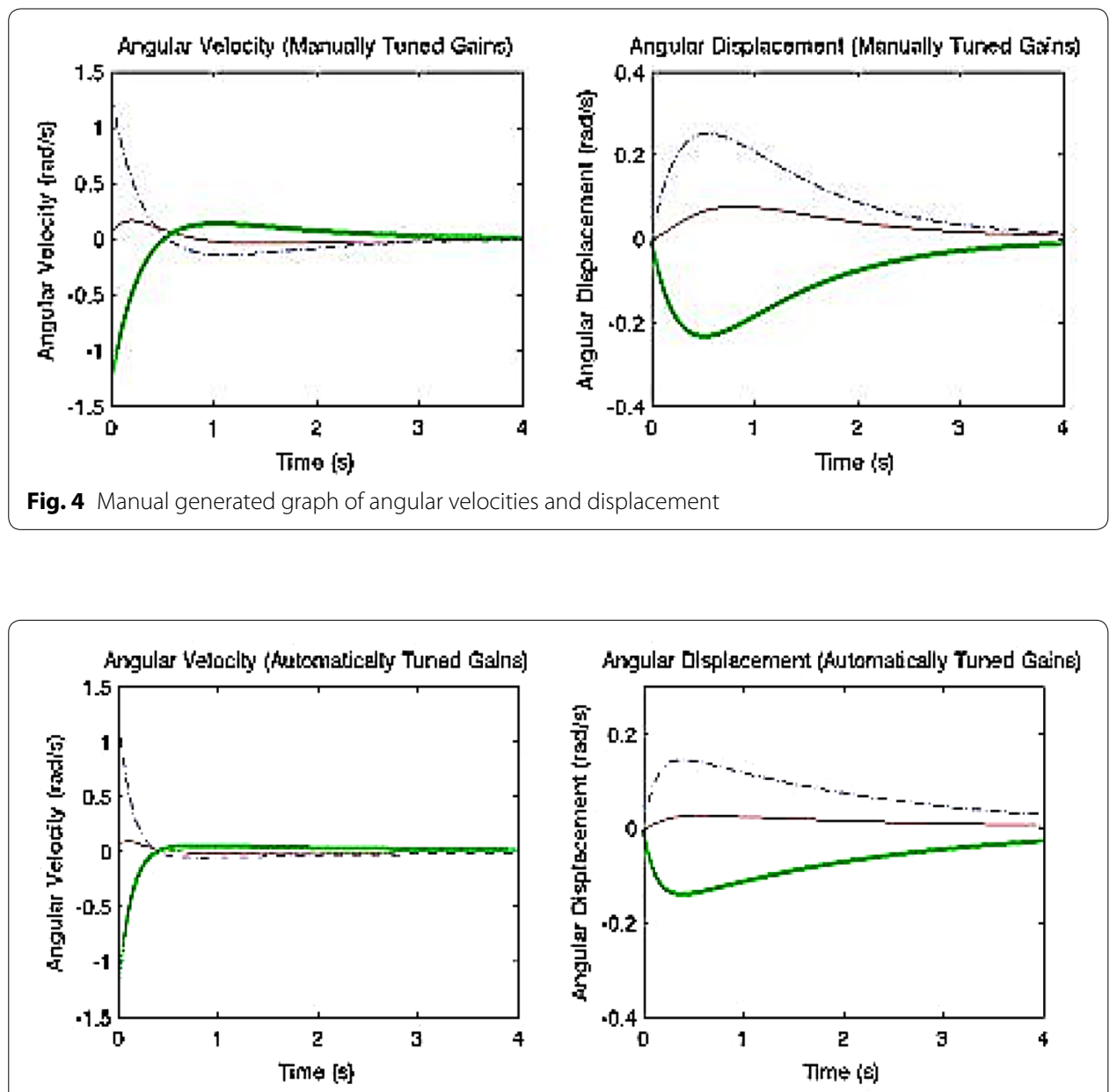

Fig. 5 Automatic generated graph of angular velocities and displacement

From the above simulated results, we inferred that the swings in the values are smaller, overshoots are lesser, and the rate of convergence is faster. The drawback in the automatically tuned angular displacement is the error which converges later then the manually tuned angular displacement.

\section{Flight dynamics}

The flight dynamics of the quadcopter generally works in the two frames. The position of the inertial frame is with respect to the ground and the gravitational pull is the negative $\mathrm{z}$-axis of the coordinates. The position of the body frame is with respect to the orientation of the quadcopter, i.e., the rotors direction are in the positive $z$-axis and the arms of the quadcopter are in $x$-axis and $y$-axis. Figure 5 shows the coordinates axis of the body and inertial frame.

The angular velocity $\omega$ is the vector quantity directing in axis of rotation $\theta$ is the time derivative of roll, pitch, and yaw. The relation below is used to determine the angular velocity vector $\omega$ in body frame where $C_{\phi}, C_{\theta}$, and $C_{\psi}$ and $S_{\phi}, S_{\theta}$, and $S_{\psi}$ represent cosine and sine angles, respectively. 


$$
\omega=\left[\begin{array}{lll}
1 & 0 & -s_{\phi} \\
0 & c_{\phi} & c_{\theta} s_{\phi} \\
0 & -s_{\phi} & c_{\theta} c_{\phi}
\end{array}\right] \dot{\theta}
$$

The relationship of the rotation matrix $\mathrm{R}$ will transform the body frame to the inertial frame. The matrix is obtained by conventions of the Euler angle, i.e., ZYZ and placing the yaw, pitch, and roll angles of the frame back to its initial values:

$$
R=\left[\begin{array}{lll}
c_{\phi} c_{\psi}-c_{\theta} s_{\phi} s_{\psi} & -c_{\psi} s_{\phi}-c_{\phi} c_{\theta} s_{\psi} & s_{\theta} s_{\psi} \\
c_{\theta} c_{\psi} s_{\phi}+c_{\phi} s_{\psi} & c_{\phi} c_{\theta} c_{\psi}-s_{\phi} s_{\psi} & -c_{\psi} s_{\theta} \\
s_{\phi} s_{\theta} & c_{\phi} s_{\theta} & c_{\theta}
\end{array}\right]
$$

The purpose of visualization of the quadcopter in three dimensions by using the mathematical model assisted in developing controllers for physical system as well as having rough idea of the rotors relative magnitude of thrust. In Fig. 6, yellow and pink bars represent the thrust magnitude on each of the four rotors.

\section{Effect of aerodynamic}

The control of the dynamics of the quadcopter at altitudes is assumed to be linear with slow speed. The varying in the speed of the air has significant effect on the dynamics of the system operating at moderate speed. Aerodynamic effect of flapping blades $[15,16]$ of the rotor affects the direction of the motion and results in the tilts of the rotor plane. To measure the flapping angle, simulated results are given in Fig. 7, which represents the increase in the horizontal force as the speed of the wind increases with time.

The type of blades used also has an effect on the aerodynamic of the system. The direction of the motion of the quadcopter frame is affected by using hinged blades (plastic material) [17] or stiff blades (carbon fiber) [17]. Figure 8 shows the change

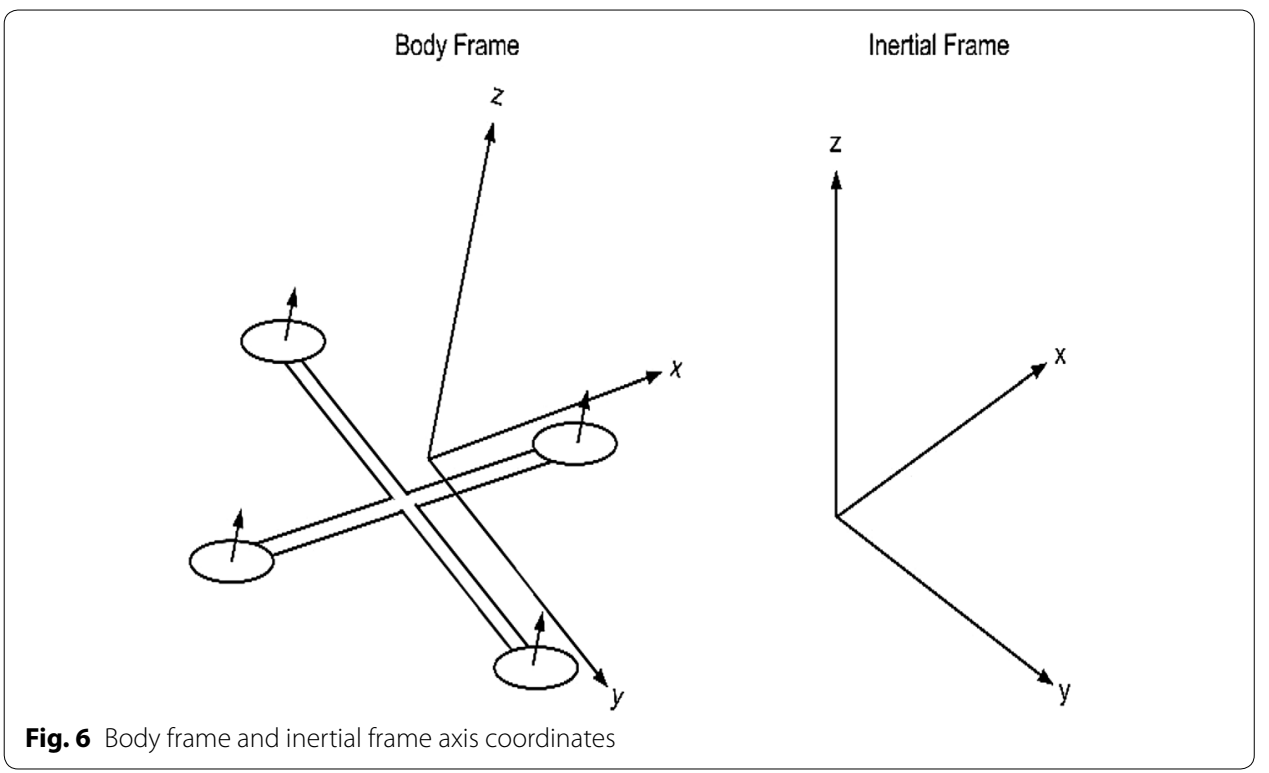




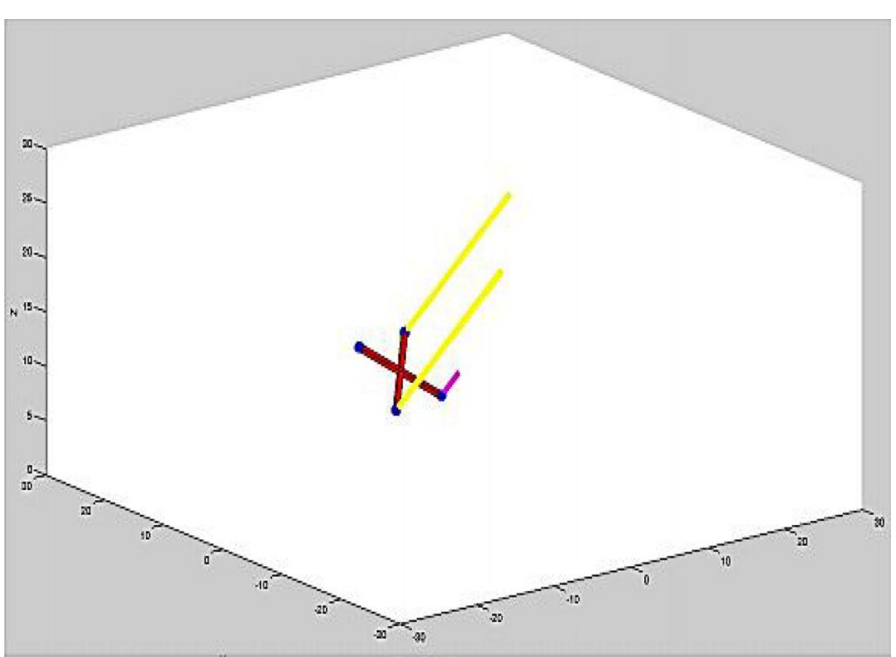

Fig. 7 Visualization of the quadcopter in three dimensions

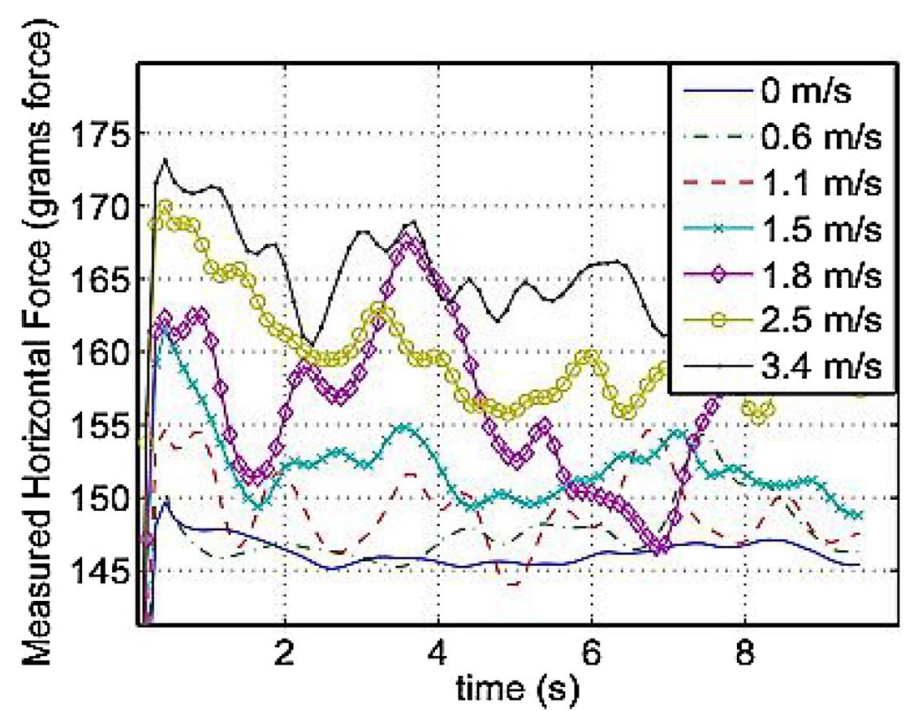

Fig. 8 Graph of the horizontal force with the increasing wind speed

in the angle of deflection versus the speed of the wind for the stiff and hinged blades. The same results were also observed during the actual flight of the quadcopter (Fig. 9).

\section{Android-based software}

The android-based software used in the project is an open source named AR drone free flight [18]. The features of the application are live video camera feed, altitude indicator, emergency landing, error reset option, quadcopter battery indicator, and joystick control. This android application is developed so that its receiver end can be any bluetooth device. We have used HC-05 bluetooth module to operate the quadcopter with this android application. 


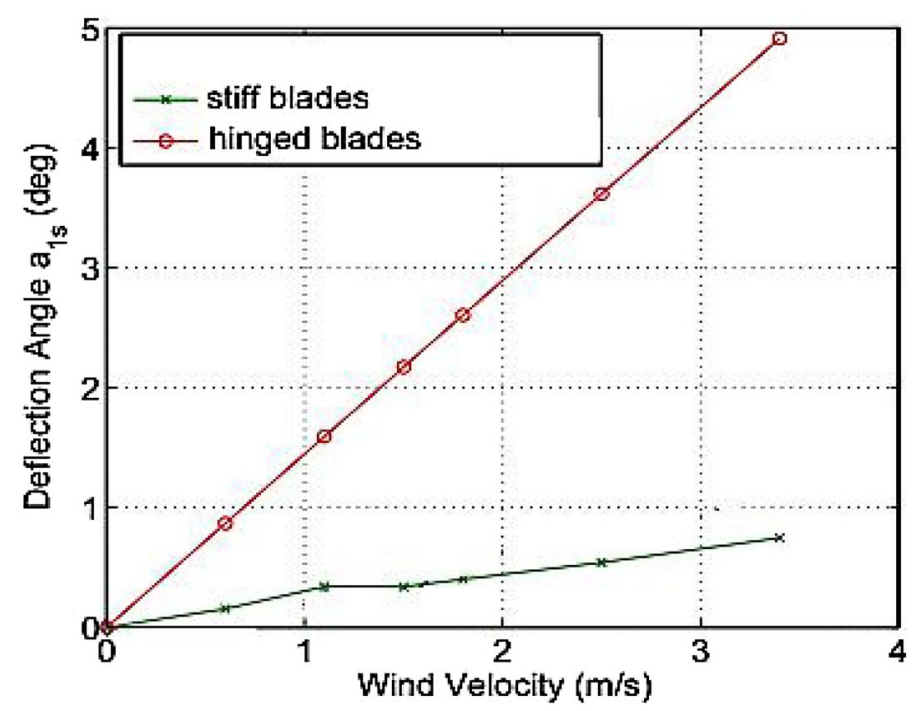

Fig. 9 Graph of the angle of deflection versus the wind speed

\section{Bluetooth device}

The bluetooth module used here is HC-05 [19], and one end of the module is serially interfaced with microcontroller. The command given from the joystick will be wirelessly communicated to the microcontroller, and quadcopter will navigate accordingly.

\section{Results}

The study of the mathematical model and control of the quadcopter is presented. Several tested methods were used for the selection of the material in the design of quadcopter frame. The rotation matrix $\mathrm{R}$ is derived from the Euler's method. The verification of the model was done by performing the simulation and implementation of the thrust magnitude on each of the rotor. Several simulation results on the aerodynamic factor of the frame and selection of the flapping blades and there effect on the angle of deflection was incorporated during the actual design. The motor behavioral modal is determined on the basis of the estimation which was quite near to the actual physical modal.

\section{Conclusion}

The stabilization factors of the quadcopter are achieved by simulating the parameters of angular velocities and angular displacement. Interfacing of the microcontroller with the brushless motors, bluetooth module, and flight controller board. Serial communication of the android-based mobile application with the bluetooth module.

\section{Abbreviations}

UAV: unmanned aerial vehicle; MEMS: microelectromechanical systems; PID: proportional integral derivative; PWM: pulse width modulation; DC: direct current; IMU: inertial measurement unit. 


\section{Authors' contributions}

SH analyzed and interpreted the mathematical modeling of the datasets regarding the flight dynamics of the quadcopter. WM performed the frame design and controller board interfacing and simulation of the quadcopter and was a major contributor in writing the manuscript. All authors read and approved the final manuscript.

\section{Funding}

Funding information is not applicable/No funding was received.

\section{Availability of data and materials}

All data generated or analyzed during the study are available in the reference section of the manuscript.

\section{Competing interests}

WM and SH authors have declare there is no competing interests.

\section{Author details}

${ }^{1}$ University of Alberta, 59127 Ave SW, Edmonton T6X0G5, Canada. ${ }^{2}$ Bahria University, Karachi, Pakistan.

Received: 21 August 2019 Accepted: 12 November 2019

Published online: 11 December 2019

\section{References}

1. Pounds P (2009) Design principles of large quad rotors for practical applications. In: ICRA, IEEE international conference, http://ieeexplore.ieee.org/document/5152390/

2. Pounds P (2009) Modelling and control of a large quad rotor robot. CEP. https://doi.org/10.1016/j.conengprac .2010 .02 .008

3. Bouabdallah S (2004) PID versus LQ control techniques applied to an indoor micro quad rotor. In: IROS/RSJ IEEE international conference, http://ieeexplore.ieee.org/document/1389776/

4. Huang H (2009) Aerodynamics and control of autonomous quad rotor helicopters in aggressive maneuvering. In: ICRA, IEEE international conference, http://ieeexplore.ieee.org/document/5152561/

5. Miller K (2008) Path tracking control for quad rotor helicopters. http://citeseerx.ist.psu.edu/viewdoc/summary?doi= 10.1.1.554.2619\&rank=1

6. Xiao J (2010) 3D indoor mapping for micro-UAVs using hybrid range finders and multi-volume occupancy grids. http://citeseerx.ist.psu.edu/viewdoc/summary?doi=10.1.1.227.7397

7. Kurtus R (2010) Explanation of the centre of gravity. School for champions, http://www.school-for champions.com/ science/gravity_center.htm\#.VipaLvmUdPO

8. Orsag M (2013) Towards the realization of mobile manipulating unmanned aerial vehicles (MM-UAV): peg-in-hole insertion tasks. In: IEEE international conference, http://ieeexplore.ieee.org/abstract/document/6556353/

9. Orsag M (2013) Mobile manipulating unmanned aerial vehicle (MM-UAV): towards aerial manipulators. In: IEEE international conference, https://pdfs.semanticscholar.org/bdad/ec99c0913f1dcfe3ab99537ee7a1651cfd9b.pdf

10. Pololu, MinIMU-9 v2, https://www.pololu.com/product/248

11. Oscar (2013) How to choose motor and propeller for quad copter and multi copter. http://blog.oscarliang.net/howto-choose-motor-and-propeller-for-quadcopter/

12. Korpela C (2012) Flight stability in aerial redundant manipulators. Robotics and automation (ICRA). In: IEEE international conference, http://ieeexplore.iee.org/document/6224925/

13. Pekala M (2013) Stability control in aerial manipulation. American Control Conference (ACC), http://ieeexplore.ieee. org/document/6580711/

14. Luukkonen T (2011) Modeling and control of quad copter,https://pdfs.semanticscholar.org/a3a8/dcfaae7afd971c2 4bd033fb0f7310e8fc741.pdf

15. Hoffmann GM (2007) Quad rotor helicopter flight dynamics and control: theory and experiment. In: Navigation and control conference and exhibit, https://arc.aiaa.org/doi/abs/10.2514/6.2007-6461

16. Huang H (2009) Aerodynamics and control of autonomous quad rotor helicopters in aggressive maneuvering. In: IEEE international conference on robotics and automation, http://ieeexplore.ieee.org/document/5152561/

17. Oscar (2014) Carbon fibre props vs plastic propeller, http://blog.oscarliang.net/carbon-fibre-props-plastic-propeller/

18. Seydoux H (2010) AR drone free flight, http://ardrone2parrot.com/

19. Rana M (2014) Smart homes for a better living using blue tooth communication based on at mega microcontroller. Int J Res Eng Technol, http://esatjournals.net/ijret/2014v03/i06/IRET20140306038.pdf

\section{Publisher's Note}

Springer Nature remains neutral with regard to jurisdictional claims in published maps and institutional affiliations. 